Article

\title{
Existence, Nonexistence and Multiplicity of Positive Solutions for Singular Boundary Value Problems Involving $\varphi$-Laplacian
}

\section{Chan-Gyun Kim it}

Department of Mathematics Education, Pusan National University, Busan 609-735, Korea; cgkim75@pusan.ac.kr or cgkim75@gmail.com

Received: 6 August 2019; Accepted: 9 October 2019; Published: 12 October 2019

Abstract: In this paper, we establish the results on the existence, nonexistence and multiplicity of positive solutions to singular boundary value problems involving $\varphi$-Laplacian. Our approach is based on the fixed point index theory. The interesting point is that a result for the existence of three positive solutions is given.

Keywords: three positive solutions; $\varphi$-Laplacian; singular problem

\section{Introduction}

In this paper, we study the existence, nonexistence and multiplicity of positive solutions to the following problem

$$
\left\{\begin{array}{l}
\left(d(t) \varphi\left(c(t) u^{\prime}\right)\right)^{\prime}+\lambda h(t) f(u)=0, t \in(0,1), \\
u(0)=u(1)=0,
\end{array}\right.
$$

where $\varphi: \mathbb{R} \rightarrow \mathbb{R}$ is an odd increasing homeomorphism, $c, d \in C([0,1],(0, \infty)), \lambda \in \mathbb{R}_{+}:=[0, \infty)$ is a parameter, $h \in C\left((0,1), \mathbb{R}_{+}\right) \backslash\{0\}$ and $f \in C\left(\mathbb{R}_{+}, \mathbb{R}_{+}\right)$with $f(s)>0$ for $s>0$.

Throughout this paper, the homeomorphism $\varphi$ satisfies the following assumption:

(A) there exist increasing homeomorphisms $\psi_{1}, \psi_{2}: \mathbb{R}_{+} \rightarrow \mathbb{R}_{+}$such that

$$
\varphi(x) \psi_{1}(y) \leq \varphi(x y) \leq \varphi(x) \psi_{2}(y) \text { for all } x, y \in \mathbb{R}_{+} .
$$

For convenience, we denote by $\mathcal{H}_{\xi}$ the set

$$
\left\{g \in C\left((0,1), \mathbb{R}_{+}\right): \int_{0}^{\frac{1}{2}} \xi^{-1}\left(\int_{s}^{\frac{1}{2}} g(\tau) d \tau\right) d s+\int_{\frac{1}{2}}^{1} \xi^{-1}\left(\int_{\frac{1}{2}}^{s} g(\tau) d \tau\right) d s<\infty\right\}
$$

where $\xi: \mathbb{R}_{+} \rightarrow \mathbb{R}_{+}$is an increasing homeomorphism and we make the following notations:

$$
f_{0}:=\lim _{s \rightarrow 0^{+}} \frac{f(s)}{\varphi(s)} \text { and } f_{\infty}:=\lim _{s \rightarrow \infty} \frac{f(s)}{\varphi(s)} .
$$

It is well known that

$$
\varphi^{-1}(x) \psi_{2}^{-1}(y) \leq \varphi^{-1}(x y) \leq \varphi^{-1}(x) \psi_{1}^{-1}(y) \text { for all } x, y \in \mathbb{R}_{+}
$$

and $L^{1}(0,1) \cap C(0,1) \subsetneq \mathcal{H}_{\psi_{1}} \subseteq \mathcal{H}_{\varphi} \subseteq \mathcal{H}_{\psi_{2}}$ (see, for example, ([1], Remark 1$)$ ). 
Problem (1) arises naturally in studying radial solutions to the following quasilinear elliptic equation defined on an annular domain

$$
\left\{\begin{array}{l}
\operatorname{div}(w(|x|) A(|\nabla v|) \nabla v)+\lambda k(|x|) f(v)=0 \text { in } \Omega, \\
v=0 \text { on } \partial \Omega
\end{array}\right.
$$

where $\Omega=\left\{x \in \mathbb{R}^{N}: R_{1}<|x|<R_{2}\right\}$ with $N \geq 2$ and $0<R_{1}<R_{2}<\infty, w \in C\left(\left[R_{1}, R_{2}\right],(0, \infty)\right)$ and $k \in C\left(\left(R_{1}, R_{2}\right), \mathbb{R}_{+}\right)$. Indeed, applying change of variables

$$
v(x)=u(t) \text { and }|x|=\left(R_{2}-R_{1}\right) t+R_{1},
$$

problem (3) is transformed into problem (1) with

$$
\varphi(s)=A(|s|) s, d(t)=w\left(\left(R_{2}-R_{1}\right) t+R_{1}\right)\left(\left(R_{2}-R_{1}\right) t+R_{1}\right)^{N-1}, c(t)=\frac{1}{R_{2}-R_{1}}
$$

and

$$
h(t)=\left(R_{2}-R_{1}\right)\left(\left(R_{2}-R_{1}\right) t+R_{1}\right)^{N-1} k\left(\left(R_{2}-R_{1}\right) t+R_{1}\right)
$$

(see, for example, References [1,2]).

For $\varphi(s)=|s|^{p-2} s$ with $p>1$, problem (1) has been extensively studied in the literature (see References [3-9] for $p=2$ and References [10-20] for $p>1$ ). For example, when $h \in \mathcal{H}_{\varphi}$ and $c \equiv d \equiv 1$, Agarwal, Lü and O'Regan [10] studied the existence and multiplicity of positive solutions to problem (1) under various assumptions on $f_{0}$ and $f_{\infty}$. In Reference [12], when $h \in \mathcal{H}_{\varphi}, c \equiv d \equiv 1$ and $f(s)$ satisfies $f(0)>0$ and $f_{\infty}=\infty$, it was shown that there exists $\lambda_{*}>0$ such that (1) has at least two positive solutions for $\lambda \in\left(0, \lambda_{*}\right)$, one positive solution for $\lambda=\lambda_{*}$ and no positive solution for $\lambda>\lambda_{*}$. Recently, under the assumptions that $h \in C^{1}((0,1],(0, \infty))$ is strictly decreasing, $h(t) \leq C t^{-\eta}$ for some $C>0$ and $\eta \in(0,1), c \equiv d \equiv 1, f \in C\left(\mathbb{R}_{+}, \mathbb{R}\right)$ is differentiable on $(0, \infty), f(0)<0$, $\limsup _{s \rightarrow 0^{+}} f^{\prime}(s)<\infty$, $f^{\prime}(s)>0$ for $s>0, \lim _{s \rightarrow \infty} f(s)=\infty, \lim _{s \rightarrow \infty} \frac{f(s)}{s^{p-1}}=0$ and $\frac{f(s)}{s^{q}}$ is non-decreasing on $[a, \infty)$ for some $a>0$ and $q \in(0, p-1)$, Shivaji, Sim and Son [19] showed the uniqueness of positive solution to problem (1) for all large $\lambda>0$. For sign-changing weight $h$ satisfying $|h| \in \mathcal{H}_{\varphi}$ and $c \equiv d \equiv 1, \mathrm{Xu}$ [20] studied the existence of a nontrivial solution to problem (1) for all small $\lambda>0$ under the assumptions that $f \in C(\mathbb{R}, \mathbb{R})$ is non-decreasing, $f(0)>0$ and $f_{\infty} \in(0, \infty)$.

For general $\varphi$ satisfying $(A)$, when $c \equiv d \equiv 1$ and $h \in L^{1}(0,1) \cap C(0,1)$, Bai and Chen [21] studied the existence of at least two positive solutions for $\lambda$ belonging to an explicit interval under some assumptions on $f$ satisfying $f(0)=0$. When $c \equiv d \equiv 1, h \in \mathcal{H}_{\psi_{1}}$ and either $f_{0}=f_{\infty}=\infty$ or $f_{0}=f_{\infty}=0$, Lee and $\mathrm{Xu}$ [22] showed that there exist $\lambda^{*} \geq \lambda_{*}>0$ such that (1) has at least two positive solutions for $\lambda \in\left(0, \lambda_{*}\right)$, one positive solution for $\lambda \in\left[\lambda_{*}, \lambda^{*}\right]$ and no positive solution for $\lambda>\lambda_{*}$. In Reference [1], for non-negative nonlinearity $f=f(t, s)$ satisfying $f\left(t_{0}, 0\right)>0$ for some $t_{0} \in[0,1]$ and $h \in \mathcal{H}_{\varphi}$, the existence of an unbounded solution component was shown and, under several assumptions on $f$ at $\infty$, the existence, nonexistence and multiplicity of positive solutions were studied.

For more general $\varphi$ which does not satisfy $(A)$, when $c \equiv d \equiv 1$ and $0 \leq h \in L^{1}(0,1)$ with $h \not \equiv 0$, Kaufmann and Milne [23] proved the existence of positive solution to problem (1) for all $\lambda>0$ under the assumptions on $f$ which induces the sublinear nonlinearity provided $\varphi(s)=|s|^{p-1} s$ with $p>1$. For other interesting results, we refer the reader to References [24-26] and the references therein.

The concavity of solutions plays a crucial role in defining a suitable cone so that the solution operator is well defined (see, for example, References [10,12,22] and the references therein). When $c \equiv d \equiv 1$, it is easy to see that solutions to problem (1) are concave functions on $[0,1]$. However, it is not clear that the solutions are concave functions on $[0,1]$, unless $c \equiv d \equiv 1$. In order to overcome this difficulty, a lemma ([2], Lemma 2.4) was proved, so that various results for the existence, nonexistence and multiplicity of positive solutions to problem (1) were proved in Reference [2] when $d$ is non-decreasing on $[0,1]$ and $h \in C[0,1]$ satisfies $h \not \equiv 0$ on any subinterval of $[0,1]$. 
The aim of this paper is to improve on the results in Reference [2] by assuming the weaker hypotheses on $h$ and $d$ than those in Reference [2]. More precisely, the monotonicity of $d$ is not assumed and the weight function $h$ may not be $L^{1}(0,1)$ and it can be vanished in some subinterval of $(0,1)$. Furthermore, a result for the existence of three positive solutions is given, which does not appear in Reference [2].

The rest of this paper is organized as follows. In Section 2, we establish preliminaries which are essential for proving our results in this paper. In Section 3, the main results are proved and an example to illustrate the results obtained in this paper is provided. Finally, the summary of this paper and future work are given in Section 4.

\section{Preliminaries}

In this section we give preliminaries which are essential for proving our results in this paper.

First, we introduce a solution operator related to problem (1). Let $g \in \mathcal{H}_{\varphi} \backslash\{0\}$ be given and define a function $v_{g}:(0,1) \rightarrow \mathbb{R}$ by

$$
v_{g}(t)=v_{g}^{1}(t)-v_{g}^{2}(t) \text { for } t \in(0,1)
$$

Here $v_{g}^{1}$ and $v_{g}^{2}$ are the functions defined by, for $t \in(0,1)$,

$$
v_{g}^{1}(t)=\int_{0}^{t} \frac{1}{c(s)} \varphi^{-1}\left(\frac{1}{d(s)} \int_{s}^{t} g(\tau) d \tau\right) d s
$$

and

$$
v_{g}^{2}(t)=\int_{t}^{1} \frac{1}{c(s)} \varphi^{-1}\left(\frac{1}{d(s)} \int_{t}^{s} g(\tau) d \tau\right) d s .
$$

It is easy to see that $v_{g}^{1}$ and $v_{g}^{2}$ are continuous and monotone functions on $(0,1)$ satisfying

$$
\lim _{t \rightarrow 0+} v_{g}^{1}(t)=\lim _{t \rightarrow 1-} v_{g}^{2}(t)=0 \text { and } \lim _{t \rightarrow 1-} v_{g}^{1}(t)=\lim _{t \rightarrow 0+} v_{g}^{2}(t) \in(0, \infty] .
$$

Thus there exists an interval $\left[\sigma_{g}^{1}, \sigma_{g}^{2}\right] \subsetneq(0,1)$ satisfying $v_{g}(\sigma)=0$ for all $\sigma \in\left[\sigma_{g}^{1}, \sigma_{g}^{2}\right]$ (see Reference [1]).

Define a function $T: \mathcal{H}_{\varphi} \rightarrow C[0,1]$ by $T(0)=0$ and, for $g \in \mathcal{H}_{\varphi} \backslash\{0\}$,

$$
T(g)(t)= \begin{cases}\int_{0}^{t} \frac{1}{c(s)} \varphi^{-1}\left(\frac{1}{d(s)} \int_{s}^{\sigma} g(\tau) d \tau\right) d s, & \text { if } 0 \leq t \leq \sigma, \\ \int_{t}^{1} \frac{1}{c(s)} \varphi^{-1}\left(\frac{1}{d(s)} \int_{\sigma}^{s} g(\tau) d \tau\right) d s, & \text { if } \sigma \leq t \leq 1 .\end{cases}
$$

where $\sigma=\sigma(g)$ is a zero of $v_{g}$ in $(0,1)$, that is,

$$
\int_{0}^{\sigma} \frac{1}{c(s)} \varphi^{-1}\left(\frac{1}{d(s)} \int_{s}^{\sigma} g(\tau) d \tau\right) d s=\int_{\sigma}^{1} \frac{1}{c(s)} \varphi^{-1}\left(\frac{1}{d(s)} \int_{\sigma}^{s} g(\tau) d \tau\right) d s .
$$

We notice that, although $\sigma=\sigma(g)$ is not necessarily unique, the right hand side of the equality in (4) does not depend on a particular choice of $\sigma$. In other words, $T(g)$ is independent of the choice of $\sigma \in\left[\sigma_{g}^{1}, \sigma_{g}^{2}\right]$ (see, for example, References [1,2]).

For $g \in \mathcal{H}_{\varphi}$, consider the following problem

$$
\left\{\begin{array}{l}
-\left(d(t) \varphi\left(c(t) u^{\prime}\right)\right)^{\prime}=g(t), \quad t \in(0,1) \\
u(0)=u(1)=0
\end{array}\right.
$$

For $g=0$, (6) has a unique zero solution due to the boundary conditions.

The usual maximum norm in a Banach space $C[0,1]$ is denoted by 


$$
\|u\|_{\infty}:=\max _{t \in[0,1]}|u(t)| \text { for } u \in C[0,1]
$$

and let

$$
\rho_{1}:=\frac{c_{0}}{\|c\|_{\infty}} \frac{\psi_{2}^{-1}\left(\frac{1}{\|d\|_{\infty}}\right)}{\psi_{1}^{-1}\left(\frac{1}{d_{0}}\right)} \in(0,1],
$$

where $c_{0}:=\min _{t \in[0,1]} c(t)>0$ and $d_{0}:=\min _{t \in[0,1]} d(t)>0$. Recently, without the monotonicity of $d$, a result similar to Reference ([2], Lemma 2.4) was proved in Reference [1].

Lemma 1 ([1], Lemma 2). Assume that $(A)$ and $g \in \mathcal{H}_{\varphi}$ hold. Then $T(g)$ is the unique solution to problem (6) and

$$
T(g)(t) \geq \min \{t, 1-t\} \rho_{1}\|T(g)\|_{\infty} \text { for } t \in[0,1] .
$$

From now on, we assume $h \in \mathcal{H}_{\varphi} \backslash\{0\}$. Let

$$
\mathcal{A}_{h}:=\{x \in(0,1): h(y)=0 \text { for all } y \in(0, x)\} \text { and } \mathcal{B}_{h}:=\{x \in(0,1): h(y)=0 \text { for all } y \in(x, 1)\} \text {. }
$$

For convenience, we use the following notations:

$\alpha_{h}:=\max \mathcal{A}_{h}$ if $\mathcal{A}_{h} \neq \varnothing$ and $\alpha_{h}:=0$ if $\mathcal{A}_{g}=\varnothing$, where $\mathcal{A}_{h}=\{x \in(0,1): h(y)=0$ for all $y \in(0, x)\}$;

$\beta_{h}:=\min \mathcal{B}_{h}$ if $\mathcal{B}_{h} \neq \varnothing$ and $\beta_{h}:=1$ if $\mathcal{B}_{g}=\varnothing$, where $\mathcal{B}_{h}=\{x \in(0,1): h(y)=0$ for all $y \in(x, 1)\} ;$

$$
\begin{gathered}
\bar{\alpha}_{h}:=\max \left\{x \in(0,1]: h(y)>0 \text { for all } y \in\left(\alpha_{h}, x\right)\right\} ; \\
\bar{\beta}_{h}:=\max \left\{x \in[0,1): h(y)>0 \text { for all } y \in\left(x, \beta_{h}\right)\right\} ; \\
\gamma_{h}^{1}:=4^{-1}\left(3 \alpha_{h}+\bar{\alpha}_{h}\right) \text { and } \gamma_{h}^{2}:=4^{-1}\left(\bar{\beta}_{h}+3 \beta_{h}\right) .
\end{gathered}
$$

Since $h \not \equiv 0$, it follows that

$$
0 \leq \alpha_{h}<\gamma_{h}^{1}<\gamma_{h}^{2}<\beta_{h} \leq 1
$$

Let $\mathcal{K}:=\left\{u \in C\left([0,1], \mathbb{R}_{+}\right): u(t) \geq \rho_{h}\|u\|_{\infty}\right.$ for $\left.t \in\left[\gamma_{h}^{1}, \gamma_{h}^{2}\right]\right\}$. Here

$$
\rho_{h}:=\rho_{1} \min \left\{\gamma_{h}^{1}, 1-\gamma_{h}^{2}\right\} \in(0,1) .
$$

Then $\mathcal{K}$ is a cone in $C[0,1]$. For $r>0$, let

$$
\mathcal{K}_{r}:=\left\{u \in \mathcal{K}:\|u\|_{\infty}<r\right\}, \partial \mathcal{K}_{r}:=\left\{u \in \mathcal{K}:\|u\|_{\infty}=r\right\} \text { and } \overline{\mathcal{K}}_{r}:=\mathcal{K}_{r} \cup \partial \mathcal{K}_{r} .
$$

Define a function $F: \mathbb{R}_{+} \times \mathcal{K} \rightarrow C(0,1)$ by

$$
F(\lambda, u)(t)=\lambda h(t) f(u(t)) \text { for }(\lambda, u) \in \mathbb{R}_{+} \times \mathcal{K} \text { and } t \in(0,1) .
$$

Clearly, $F(\lambda, u) \in \mathcal{H}_{\varphi}$ for any $(\lambda, u) \in \mathbb{R}_{+} \times \mathcal{K}$.

Now we define an operator $H: \mathbb{R}_{+} \times \mathcal{K} \rightarrow C[0,1]$ by

$$
H(\lambda, u) \equiv T(F(\lambda, u)) \text { for }(\lambda, u) \in \mathbb{R}_{+} \times \mathcal{K} .
$$

That is, for $(\lambda, u) \in \mathbb{R}_{+} \times \mathcal{K}$,

$$
H(\lambda, u)(t)= \begin{cases}\int_{0}^{t} \frac{1}{c(s)} \varphi^{-1}\left(\frac{1}{d(s)} \int_{s}^{\sigma} F(\lambda, u)(\tau) d \tau\right) d s, & \text { if } 0 \leq t \leq \sigma, \\ \int_{t}^{1} \frac{1}{c(s)} \varphi^{-1}\left(\frac{1}{d(s)} \int_{\sigma}^{s} F(\lambda, u)(\tau) d \tau\right) d s, & \text { if } \sigma \leq t \leq 1,\end{cases}
$$

where $\sigma=\sigma(\lambda, u)$ is a number satisfying

$$
\int_{0}^{\sigma} \frac{1}{c(s)} \varphi^{-1}\left(\frac{1}{d(s)} \int_{s}^{\sigma} F(\lambda, u)(\tau) d \tau\right) d s=\int_{\sigma}^{1} \frac{1}{c(s)} \varphi^{-1}\left(\frac{1}{d(s)} \int_{\sigma}^{s} F(\lambda, u)(\tau) d \tau\right) d s .
$$




\section{Remark 1.}

(1) For any $(\lambda, u) \in \mathbb{R}_{+} \times \mathcal{K}$, by Lemma $1, H\left(\mathbb{R}_{+} \times \mathcal{K}\right) \subseteq \mathcal{K}$.

(2) It is easy to see that (1) has a solution if and only if $H(\lambda, \cdot)$ has a fixed point in $\mathcal{K}$.

(3) Since $H(0, u)=0$ for all $u \in \mathcal{K}, 0$ is a unique solution to problem (1) with $\lambda=0$. By Lemma 1, any nonzero solution $u$ to problem (1) is positive one, that is, $u(t)>0$ for all $t \in(0,1)$.

By the argument similar to those in the proof of ([10], Lemma 3), it can be proved that $H$ is completely continuous on $\mathbb{R}_{+} \times \mathcal{K}$ (see also Reference ([27], Lemma 3.3)). We omit the proof of it.

Lemma 2 ([1], Lemma 4). Assume that $(A)$ and $g \in \mathcal{H}_{\varphi}$ hold. Then the operator $H: \mathbb{R}_{+} \times \mathcal{K} \rightarrow \mathcal{K}$ is completely continuous, that is, compact and continuous.

Finally, we recall a well-known theorem of the fixed point index theory.

Theorem 1 (see, for example, References [28,29]). Assume that, for some $r>0, T_{1}: \overline{\mathcal{K}}_{r} \rightarrow \mathcal{K}$ is completely continuous on $\overline{\mathcal{K}}_{r}$. Then

$$
\begin{aligned}
& \text { (i) if }\left\|T_{1}(u)\right\|_{\infty}>\|u\|_{\infty} \text { for } u \in \partial \mathcal{K}_{r} \text {, then } i\left(T_{1}, \mathcal{K}_{r}, \mathcal{K}\right)=0 \\
& \text { (ii) if }\left\|T_{1}(u)\right\|_{\infty}<\|u\|_{\infty} \text { for } u \in \partial \mathcal{K}_{r} \text {, then } i\left(T_{1}, \mathcal{K}_{r}, \mathcal{K}\right)=1
\end{aligned}
$$

\section{Main Results}

Throughout this section, we assume $h \in \mathcal{H}_{\psi_{1}} \backslash\{0\}$. For convenience, we use the following notations in this section:

$$
\begin{gathered}
\gamma_{h}:=\frac{\gamma_{h}^{1}+\gamma_{h}^{2}}{2}, \text { where } \gamma_{h}^{1}=\frac{3 \alpha_{h}+\bar{\alpha}_{h}}{4} \text { and } \gamma_{h}^{2}=\frac{\bar{\beta}_{h}+3 \beta_{h}}{4} \\
A_{1}:=\frac{1}{\|c\|_{\infty}} \psi_{2}^{-1}\left(\frac{1}{\|d\|_{\infty}}\right) \min \left\{\int_{\gamma_{h}^{1}}^{\gamma_{h}} \psi_{2}^{-1}\left(\int_{s}^{\gamma_{h}} h(\tau) d \tau\right) d s, \int_{\gamma_{h}}^{\gamma_{h}^{2}} \psi_{2}^{-1}\left(\int_{\gamma_{h}}^{s} h(\tau) d \tau\right) d s\right\} ; \\
A_{2}:=\frac{1}{c_{0}} \psi_{1}^{-1}\left(\frac{1}{d_{0}}\right) \max \left\{\int_{0}^{\gamma_{h}} \psi_{1}^{-1}\left(\int_{s}^{\gamma_{h}} h(\tau) d \tau\right) d s, \int_{\gamma_{h}}^{1} \psi_{1}^{-1}\left(\int_{\gamma_{h}}^{s} h(\tau) d \tau\right) d s\right\} .
\end{gathered}
$$

Since $0 \leq \alpha_{h}<\gamma_{h}^{1}<\gamma_{h}<\gamma_{h}^{2}<\beta_{h} \leq 1, A_{1}>0$ and $A_{2}>0$. Define continuous functions $R_{1}, R_{2}:(0, \infty) \rightarrow(0, \infty)$ by

$$
R_{1}(m):=\frac{1}{f_{*}(m)} \varphi\left(\frac{m}{A_{1}}\right) \text { and } R_{2}(m):=\frac{1}{f^{*}(m)} \varphi\left(\frac{m}{A_{2}}\right) \text { for } m \in(0, \infty) .
$$

Here, $f_{*}(m):=\min \left\{f(y): \rho_{h} m \leq y \leq m\right\}$ and $f^{*}(m):=\max \{f(y): 0 \leq y \leq m\}$ for $m \in \mathbb{R}_{+}$.

\section{Remark 2.}

(1) By (2), $\psi_{2}^{-1}(y) \leq \psi_{1}^{-1}(y)$ for all $y \in \mathbb{R}_{+}$, and it follows that

$$
0<A_{1}<A_{2} \text { and } 0<R_{2}(m)<R_{1}(m) \text { for all } m \in(0, \infty) \text {. }
$$

(2) For any $l \in C\left(\mathbb{R}_{+}, \mathbb{R}_{+}\right), l_{a}:=\lim _{m \rightarrow a} \frac{l(m)}{\varphi(m)}$ for $a \in\{0, \infty\}$. It is not hard to prove that

$$
\left(f_{*}\right)_{a}=\left(f^{*}\right)_{a}=0 \text { if } f_{a}=0 \text { and }\left(f_{*}\right)_{a}=\left(f^{*}\right)_{a}=\infty \text { if } f_{a}=\infty .
$$

For convenience of readers, we give the proofs. First, we show that $f_{0}=0$ implies $\left(f_{*}\right)_{0}=\left(f^{*}\right)_{0}=0$. Let $\epsilon>0$ be given and $f_{0}=0$ be assumed. Then there exists $\delta>0$ such that for any $s \in(0, \delta)$, $0<\frac{f(s)}{\varphi(s)}<\epsilon$. Since $f \in C\left(\mathbb{R}_{+}, \mathbb{R}_{+}\right)$with $f(s)>0$ for $s \in(0, \infty)$, by the extreme value theorem, for any $s \in(0, \infty), f^{*}(s)=f\left(x_{s}\right)$ for some $x_{s} \in(0, s]$. Then 


$$
0 \leq \frac{f_{*}(s)}{\varphi(s)} \leq \frac{f^{*}(s)}{\varphi(s)}=\frac{f\left(x_{s}\right)}{\varphi(s)} \leq \frac{f\left(x_{s}\right)}{\varphi\left(x_{s}\right)}<\epsilon \text { for any } s \in(0, \delta)
$$

which implies that $\left(f_{*}\right)_{0}=\left(f^{*}\right)_{0}=0$.

Next, we show that $f_{\infty}=0$ implies $\left(f_{*}\right)_{\infty}=\left(f^{*}\right)_{\infty}=0$. Indeed, let $\epsilon>0$ be given and $f_{\infty}=0$ be assumed. Then there exists $N>0$ such that for any $s \geq N, \frac{f(s)}{\varphi(s)}<\epsilon$. For $s \geq N, f^{*}(s) \leq$ $f^{*}(N)+f\left(x_{N, s}\right)$, where $x_{N, s}$ is the point in $[N, s]$ satisfying $f\left(x_{N, s}\right)=\max \{f(x): N \leq x \leq s\}$. Then

$$
0 \leq \frac{f_{*}(s)}{\varphi(s)} \leq \frac{f^{*}(s)}{\varphi(s)} \leq \frac{f^{*}(N)}{\varphi(s)}+\frac{f^{*}\left(x_{N, s}\right)}{\varphi\left(x_{N, s}\right)} \leq \frac{f^{*}(N)}{\varphi(s)}+\epsilon .
$$

Consequently, $0 \leq \limsup _{s \rightarrow \infty} \frac{f_{*}(s)}{\varphi(s)} \leq \limsup _{s \rightarrow \infty} \frac{f^{*}(s)}{\varphi(s)} \leq \epsilon$, which is true for all $\epsilon>0$. Thus $\left(f_{*}\right)_{\infty}=$ $\left(f^{*}\right)_{\infty}=0$.

Finally, we show that, for $a \in\{0, \infty\}, f_{a}=\infty$ implies $\left(f_{*}\right)_{a}=\infty$. For each $m \in(0, \infty)$, by the extreme value theorem, there exists $m_{*} \in\left[\rho_{h} m, m\right]$ satisfying $\left(f_{*}\right)(m)=f\left(m_{*}\right)$, and

$$
\frac{f_{*}(m)}{\varphi(m)}=\frac{f\left(m_{*}\right)}{\varphi(m)} \geq \frac{f\left(m_{*}\right)}{\varphi\left(m_{*}\right) \psi_{2}\left(\frac{m}{m_{*}}\right)} \geq \frac{f\left(m_{*}\right)}{\varphi\left(m_{*}\right) \psi_{2}\left(\rho_{h}^{-1}\right)} .
$$

As $m \rightarrow a \in\{0, \infty\}, m_{*} \rightarrow a$ and thus $\left(f_{*}\right)_{a}=\infty$, provided $f_{a}=\infty$.

(3) By $(A)$ and Remark $2(2)$, for $i \in\{1,2\}$ and $a \in\{0, \infty\}$,

$$
\lim _{m \rightarrow a} R_{i}(m)=\infty \text { if } f_{a}=0 \text { and } \lim _{m \rightarrow a} R_{i}(m)=0 \text { if } f_{a}=\infty
$$

Lemma 3. Assume that $(A)$ and $h \in \mathcal{H}_{\psi_{1}} \backslash\{0\}$ hold. Let $m \in(0, \infty)$ be fixed. Then, for any $\lambda \in\left(R_{1}(m), \infty\right)$, $\|H(\lambda, v)\|_{\infty}>\|v\|$ for all $v \in \partial \mathcal{K}_{m}$ and

$$
i\left(H(\lambda, \cdot), \mathcal{K}_{m}, \mathcal{K}\right)=0
$$

Proof. Let $\lambda \in\left(R_{1}(m), \infty\right)$ and $v \in \partial \mathcal{K}_{m}$ be fixed. Then $\rho_{h} m \leq v(t) \leq m$ for $t \in\left[\gamma_{h}^{1}, \gamma_{h}^{2}\right]$, and

$$
f(v(t)) \geq f_{*}(m)=\frac{1}{R_{1}(m)} \varphi\left(\frac{m}{A_{1}}\right) \text { for } t \in\left[\gamma_{h}^{1}, \gamma_{h}^{2}\right]
$$

Let $\sigma$ be a number satisfying $H(\lambda, v)(\sigma)=\|H(\lambda, v)\|_{\infty}$. We have two cases: either $(i) \sigma \in\left[\gamma_{h}, 1\right)$ or $\left(\right.$ ii) $\sigma \in\left(0, \gamma_{h}\right)$. We only consider the case $(i)$, since the case (ii) can be dealt in a similar manner. Since $\lambda>R_{1}(m)$, it follows from (2) and (9) that

$$
\begin{aligned}
\|H(\lambda, v)\|_{\infty} & =\int_{0}^{\sigma} \frac{1}{c(s)} \varphi^{-1}\left(\frac{1}{d(s)} \int_{s}^{\sigma} \lambda h(\tau) f(v(\tau)) d \tau\right) d s \\
& \geq \frac{1}{\|c\|_{\infty}} \int_{\gamma_{h}^{1}}^{\gamma_{h}} \varphi^{-1}\left(\int_{s}^{\gamma_{h}} h(\tau) d \tau \frac{\lambda}{\|d\|_{\infty} R_{1}(m)} \varphi\left(\frac{m}{A_{1}}\right)\right) d s \\
& \geq \frac{1}{\|c\|_{\infty}} \int_{\gamma_{h}^{1}}^{\gamma_{h}} \psi_{2}^{-1}\left(\int_{s}^{\gamma_{h}} h(\tau) d \tau\right) d s \varphi^{-1}\left(\frac{\lambda}{\|d\|_{\infty} R_{1}(m)} \varphi\left(\frac{m}{A_{1}}\right)\right) \\
& >\frac{1}{\|c\|_{\infty}} \int_{\gamma_{h}^{1}}^{\gamma_{h}} \psi_{2}^{-1}\left(\int_{s}^{\gamma_{h}} h(\tau) d \tau\right) d s \psi_{2}^{-1}\left(\frac{1}{\|d\|_{\infty}}\right) \frac{m}{A_{1}} \geq m=\left\|_{\infty}\right\|_{\infty} .
\end{aligned}
$$

By Theorem 1, (8) holds for any $\lambda \in\left(R_{1}(m), \infty\right)$. 
Lemma 4. Assume that $(A)$ and $h \in \mathcal{H}_{\psi_{1}} \backslash\{0\}$ hold. Let $m \in(0, \infty)$ be fixed. Then, for any $\lambda \in\left(0, R_{2}(m)\right)$, $\|H(\lambda, v)\|_{\infty}<\|v\|$ for all $v \in \partial \mathcal{K}_{m}$ and

$$
i\left(H(\lambda, \cdot), \mathcal{K}_{m}, \mathcal{K}\right)=1
$$

Proof. Let $\lambda \in\left(0, R_{2}(m)\right)$ and $v \in \partial \mathcal{K}_{m}$ be fixed. Then $f(v(t)) \leq \frac{1}{R_{2}(m)} \varphi\left(\frac{m}{A_{2}}\right)$ for $t \in[0,1]$. By the same argument as in the proof of Lemma 3, it follows that $\|H(\lambda, v)\|_{\infty}<\|v\|$ for all $v \in \partial \mathcal{K}_{m}$ and (10) holds for any $\lambda \in\left(0, R_{2}(m)\right)$.

By Lemmas 3 and 4, we give some results for the existence and multiplicity of positive solutions to problem (1). Since the proofs are similar, we only give the proof of Theorem 2.

Theorem 2. Assume that $(A)$ and $h \in \mathcal{H}_{\psi_{1}} \backslash\{0\}$ hold and that there exist $m_{1}$ and $m_{2}$ such that $0<m_{1}<m_{2}$ (resp., $\left.0<m_{2}<m_{1}\right)$ and $R_{1}\left(m_{1}\right)<R_{2}\left(m_{2}\right)$. Then (1) has a positive solution $u=u(\lambda)$ satisfying $m_{1}<\|u\|_{\infty}<m_{2}$ (resp., $\left.m_{2}<\|u\|_{\infty}<m_{1}\right)$ for any $\lambda \in\left(R_{1}\left(m_{1}\right), R_{2}\left(m_{2}\right)\right)$.

Proof. We only prove the case $0<m_{1}<m_{2}$, since the other case is similar. Let $\lambda \in\left(R_{1}\left(m_{1}\right), R_{2}\left(m_{2}\right)\right)$ be fixed. By Lemmas 3 and 4 ,

$$
i\left(H(\lambda, \cdot), \mathcal{K}_{m_{1}}, \mathcal{K}\right)=1 \text { and } i\left(H(\lambda, \cdot), \mathcal{K}_{m_{2}}, \mathcal{K}\right)=0 .
$$

Since $H(\lambda, v) \neq v$ for all $v \in \partial \mathcal{K}_{m_{1}}$, it follows from the additivity property that

$$
i\left(H(\lambda, \cdot), \mathcal{K}_{m_{2}} \backslash \overline{\mathcal{K}}_{m_{1}}, \mathcal{K}\right)=-1 .
$$

Then there exists $u \in \mathcal{K}_{m_{2}} \backslash \overline{\mathcal{K}}_{m_{1}}$ such that $H(\lambda, u)=u$ by the solution property. Thus the proof is complete.

For Theorems 3 and 4, let

$$
R_{*}:=\max \left\{R_{1}\left(m_{1}\right), R_{1}\left(M_{1}\right)\right\} \text { and } R^{*}:=\min \left\{R_{2}\left(m_{2}\right), R_{2}\left(M_{2}\right)\right\} .
$$

Theorem 3. Assume that $(A)$ and $h \in \mathcal{H}_{\psi_{1}} \backslash\{0\}$ hold and that there exist $m_{1}, m_{2}$ and $M_{1}$ (resp., $M_{2}$ ) such that $0<m_{1}<m_{2}<M_{1}$ (resp., $0<m_{2}<m_{1}<M_{2}$ ) and $R_{*}<R_{2}\left(m_{2}\right)$ (resp., $\left.R_{1}\left(m_{1}\right)<R^{*}\right)$. Then (1) has two positive solutions $u_{1}=u_{1}(\lambda)$ and $u_{2}=u_{2}(\lambda)$ satisfying $m_{1}<\left\|u_{1}\right\|_{\infty}<m_{2}<\left\|u_{2}\right\|_{\infty}<M_{1}$ for any $\lambda \in\left(R_{*}, R_{2}\left(m_{2}\right)\right)$ (resp., $m_{2}<\left\|u_{1}\right\|_{\infty}<m_{1}<\left\|u_{2}\right\|_{\infty}<M_{2}$ for any $\lambda \in\left(R_{1}\left(m_{1}\right), R^{*}\right)$ ).

Theorem 4. Assume that $(A)$ and $h \in \mathcal{H}_{\psi_{1}} \backslash\{0\}$ hold and that there exist $m_{1}, m_{2}, M_{1}$ and $M_{2}$ such that $0<$ $m_{2}<m_{1}<M_{2}<M_{1}$ (resp., $0<m_{1}<m_{2}<M_{1}<M_{2}$ ) and $R_{*}<R^{*}$. Then (1) has three positive solutions $u_{1}=u_{1}(\lambda), u_{2}=u_{2}(\lambda)$ and $u_{3}=u_{3}(\lambda)$ satisfying $m_{2}<\left\|u_{1}\right\|_{\infty}<m_{1}<\left\|u_{2}\right\|_{\infty}<M_{2}<\left\|u_{3}\right\|_{\infty}<M_{1}$ (resp., $\left.m_{1}<\left\|u_{1}\right\|_{\infty}<m_{2}<\left\|u_{2}\right\|_{\infty}<M_{1}<\left\|u_{3}\right\|_{\infty}<M_{2}\right)$ for any $\lambda \in\left(R_{*}, R^{*}\right)$.

Now we give the existence and nonexistence results for positive solutions to problem (1) which are analogous to Reference ([2], Theorems 1.1 and 1.2).

Theorem 5. Assume that $(A)$ and $h \in \mathcal{H}_{\psi_{1}} \backslash\{0\}$ hold.

(1) If $f_{0}=0$ and $f_{\infty}=\infty$ (resp., $f_{0}=\infty$ and $f_{\infty}=0$ ), then (1) has a positive solution $u(\lambda)$ for any $\lambda \in(0, \infty)$ satisfying $\left\|u_{\lambda}\right\|_{\infty} \rightarrow \infty$ as $\lambda \rightarrow 0$ and $\left\|u_{\lambda}\right\|_{\infty} \rightarrow 0$ as $\lambda \rightarrow \infty$ (resp., $\left\|u_{\lambda}\right\|_{\infty} \rightarrow 0$ as $\lambda \rightarrow 0$ and $\left\|u_{\lambda}\right\|_{\infty} \rightarrow \infty$ as $\left.\lambda \rightarrow \infty\right)$.

(2) If $f_{0}=0$ and $f_{\infty} \in(0, \infty)$ (resp., $f_{0} \in(0, \infty)$ and $f_{\infty}=0$ ), then there exists $\lambda_{*} \in(0, \infty)$ and $m_{*} \in(0, \infty]$ (resp., $\left.m_{*} \in \mathbb{R}_{+}\right)$such that (1) has a positive solution $u(\lambda)$ for any $\lambda \in\left(\lambda_{*}, \infty\right)$ satisfying $\|u(\lambda)\|_{\infty} \in\left(0, m_{*}\right)$ and $\lim _{\lambda \rightarrow \infty}\|u(\lambda)\|_{\infty}=0$ (resp., $\|u(\lambda)\|_{\infty} \in\left(m_{*}, \infty\right)$ and $\lim _{\lambda \rightarrow \infty}\|u(\lambda)\|_{\infty}=\infty$ ). Moreover, if $m_{*} \in(0, \infty)$, then there exists a positive solution $u\left(\lambda_{*}\right)$ to problem (1) with $\lambda=\lambda_{*}$. 
(3) If $f_{0}=\infty$ and $f_{\infty} \in(0, \infty)$ (resp., $f_{0} \in(0, \infty)$ and $\left.f_{\infty}=\infty\right)$, then there exist $\lambda^{*} \in(0, \infty)$ and $m^{*} \in(0, \infty]$ (resp., $m^{*} \in \mathbb{R}_{+}$) such that problem (1) has a positive solution $u=u(\lambda)$ for any $\lambda \in\left(0, \lambda^{*}\right)$ satisfying $\|u(\lambda)\|_{\infty} \in\left(0, m^{*}\right)$ and $\lim _{\lambda \rightarrow 0}\|u(\lambda)\|_{\infty}=0$ (resp., $\|u(\lambda)\|_{\infty} \in\left(m^{*}, \infty\right)$ and $\lim _{\lambda \rightarrow 0}\|u(\lambda)\|_{\infty}=$ $\infty)$. Moreover, if $m^{*} \in(0, \infty)$, then there exists a positive solution $u\left(\lambda^{*}\right)$ to problem $(1)$ with $\lambda=\lambda^{*}$.

(4) If $f_{0}=f_{\infty}=0$, then there exist $\lambda_{*} \in(0, \infty)$ and $m_{*} \in(0, \infty)$ such that (1) has two positive solutions $u_{1}(\lambda)$ and $u_{2}(\lambda)$ for any $\lambda \in\left(\lambda_{*}, \infty\right)$ and it has a positive solution $u\left(\lambda_{*}\right)$ for $\lambda=\lambda_{*}$. Moreover, $u_{1}(\lambda)$ and $u_{2}(\lambda)$ can be chosen so that $0<\left\|u_{1}(\lambda)\right\|_{\infty}<m_{*}<\left\|u_{2}(\lambda)\right\|_{\infty}, \lim _{\lambda \rightarrow \infty}\left\|u_{1}(\lambda)\right\|_{\infty}=0$ and $\lim _{\lambda \rightarrow \infty}\left\|u_{2}(\lambda)\right\|_{\infty}=\infty$.

(5) If $f_{0}=f_{\infty}=\infty$, then there exist $\lambda^{*} \in(0, \infty)$ and $m^{*} \in(0, \infty)$ such that problem (1) has two positive solutions $u_{1}(\lambda)$ and $u_{2}(\lambda)$ for any $\lambda \in\left(0, \lambda^{*}\right)$ and it has a positive solution $u\left(\lambda^{*}\right)$ for $\lambda=\lambda^{*}$. Moreover, $u_{1}(\lambda)$ and $u_{2}(\lambda)$ can be chosen so that $0<\left\|u_{1}(\lambda)\right\|_{\infty}<m^{*}<\left\|u_{2}(\lambda)\right\|_{\infty}, \lim _{\lambda \rightarrow 0}\left\|u_{1}(\lambda)\right\|_{\infty}=0$ and $\lim _{\lambda \rightarrow 0}\left\|u_{2}(\lambda)\right\|_{\infty}=\infty$

(6) If $f_{0} \in[0, \infty)$ and $f_{\infty} \in[0, \infty)$, then there exists $\bar{\lambda}>0$ such that (1) has no positive solutions for $\lambda<\bar{\lambda}$.

(7) If $f_{0} \in(0, \infty]$ and $f_{\infty} \in(0, \infty]$, then there exists $\underline{\lambda}>0$ such that (1) has no positive solutions for $\lambda>\underline{\lambda}$.

Proof. (1) We only give the proof of the case $f_{0}=0$ and $f_{\infty}=\infty$, since the case $f_{0}=\infty$ and $f_{\infty}=0$ can be proved in a similar manner. Since $f_{0}=0$ and $f_{\infty}=\infty$, by Remark 2 (3), $R_{i}(m) \rightarrow \infty$ as $m \rightarrow 0$ and $R_{i}(m) \rightarrow 0$ as $m \rightarrow \infty$ for $i=1,2$. For any $\lambda \in(0, \infty)$, there exist $m_{1}(\lambda)$ and $m_{2}(\lambda)$ such that

$$
0<m_{2}(\lambda)<m_{1}(\lambda) \text { and } R_{1}\left(m_{1}(\lambda)\right)<\lambda<R_{2}\left(m_{2}(\lambda)\right) .
$$

By Theorem 2, there exists a positive solution $u_{\lambda}$ to problem (1) satisfying $m_{2}(\lambda)<\left\|u_{\lambda}\right\|_{\infty}<$ $m_{1}(\lambda)$. Since $R_{i}(m) \rightarrow \infty$ as $m \rightarrow 0$ for $i=1,2$, we may choose $m_{1}(\lambda)$ and $m_{2}(\lambda)$ so that $0<m_{2}(\lambda)<$ $m_{1}(\lambda)$ and $m_{1}(\lambda) \rightarrow 0$ as $\lambda \rightarrow \infty$. Consequently, we can choose positive solutions $u_{\lambda}$ to problem (1) for large $\lambda>0$ so that $\left\|u_{\lambda}\right\|_{\infty} \rightarrow 0$ as $\lambda \rightarrow \infty$. Similarly, since $R_{i}(m) \rightarrow 0$ as $m \rightarrow \infty$ for $i=1,2$, we can choose positive solutions $u_{\lambda}$ to problem (1) for small $\lambda>0$ so that $\left\|u_{\lambda}\right\|_{\infty} \rightarrow \infty$ as $\lambda \rightarrow 0$.

(2) We only give the proof of the case $f_{0}=0$ and $f_{\infty} \in(0, \infty)$, since the case $f_{0} \in(0, \infty)$ and $f_{\infty}=0$ can be proved in a similar manner. Since $f_{0}=0$, by Remark $2(3), R_{i}(m) \rightarrow \infty$ as $m \rightarrow 0$ for $i=1$, 2 . Since

$$
\lim _{m \rightarrow \infty} R_{1}(m) \geq \lim _{m \rightarrow \infty} \frac{\varphi\left(\frac{m}{A_{1}}\right)}{f(m)} \geq \lim _{m \rightarrow \infty} \frac{\varphi(m)}{f(m)} \psi_{1}\left(\frac{1}{A_{1}}\right)=\frac{1}{f_{\infty}} \psi_{1}\left(\frac{1}{A_{1}}\right)>0,
$$

there exist $\lambda_{*}:=\inf \left\{R_{1}(m): m \in(0, \infty)\right\} \in(0, \infty)$ and $m_{*} \in(0, \infty]$ satisfying $R_{1}\left(m_{*}\right)=\lambda_{*}$. For any $\lambda \in\left(\lambda_{*}, \infty\right)$, there exist $m_{1}(\lambda)$ and $m_{2}(\lambda)$ such that

$$
0<m_{2}(\lambda)<m_{1}(\lambda)<m_{*} \text { and } R_{1}\left(m_{1}\right)<\lambda<R_{2}\left(m_{2}\right) .
$$

By Theorem 2, there exists a positive solution $u_{\lambda}$ to problem (1) satisfying $m_{2}(\lambda)<\|u\|_{\infty}<m_{1}(\lambda)$. Since $R_{i}(m) \rightarrow \infty$ as $m \rightarrow 0$ for $i=1,2$, we may choose $m_{1}(\lambda)$ and $m_{2}(\lambda)$ satisfying $0<m_{2}(\lambda)<m_{1}(\lambda)$ and $m_{1}(\lambda) \rightarrow 0$ as $\lambda \rightarrow \infty$. Consequently, we can choose positive solutions $u_{\lambda}$ to problem (1) for large $\lambda>0$ so that $\left\|u_{\lambda}\right\|_{\infty} \rightarrow 0$ as $\lambda \rightarrow \infty$.

For each $n \in \mathbb{N}$, let $\lambda_{n}:=\lambda_{*}+\frac{1}{n}$. Then we may choose $m_{1}=m_{1}(n)$ and $m_{2}=m_{2}(n)$ such that

$$
R_{1}\left(m_{1}(n)\right)<\lambda_{n}<R_{2}\left(m_{2}(n)\right) \text { and } 0<\delta<m_{2}(n)<m_{1}(n)<m_{*} \text { for all } n \text {. }
$$

Consequently, for each $n$, there exists $u_{n} \in \mathcal{K}$ such that $H\left(\lambda_{n}, u_{n}\right)=u_{n}$ and $\delta<\left\|u_{n}\right\|_{\infty}<m_{*}$. Since $\left\{\left(\lambda_{n}, u_{n}\right)\right\}$ is bounded in $\mathbb{R}_{+} \times \mathcal{K}$ and $H: \mathbb{R}_{+} \times \mathcal{K} \rightarrow \mathcal{K}$ is compact, there exist a subsequence $\left\{\left(\lambda_{n_{k}}, u_{n_{k}}\right)\right\}$ of $\left\{\left(\lambda_{n}, u_{n}\right)\right\}$ and $u_{*} \in \mathcal{K}$ such that $H\left(\lambda_{n_{k}}, u_{n_{k}}\right)=u_{n_{k}} \rightarrow u_{*}$ in $\mathcal{K}$ as $k \rightarrow \infty$. Since $\lambda_{n} \rightarrow \lambda_{*}$ as $n \rightarrow \infty$ and $H$ is continuous, $H\left(\lambda_{*}, u_{*}\right)=u_{*}$ and $\left\|u_{*}\right\|_{\infty} \geq \delta>0$. Thus problem (1) has a positive solution $u_{*}$ for $\lambda=\lambda_{*}$. Thus the proof is complete.

(3) Let $\lambda^{*}=\sup \left\{R_{2}(m): m \in(0, \infty)\right\} \in(0, \infty)$ and $m^{*} \in[0, \infty]$ satisfying $R_{2}\left(m^{*}\right)=\lambda^{*}$. Then the proof is complete by the argument similar to those in the proof of Theorem 5 (2). 
(4) Since $f_{0}=f_{\infty}=0$, it follows that, for $i=1,2, \lim _{m \rightarrow 0} R_{i}(m)=\lim _{m \rightarrow \infty} R_{i}(m)=\infty$. Then there exists $m_{*} \in(0, \infty)$ satisfying $R_{1}\left(m_{*}\right)=\min \left\{R_{1}(m): m \in \mathbb{R}_{+}\right\} \in(0, \infty)$. Let $\lambda_{*}=R_{1}\left(m_{*}\right)$. For any $\lambda \in\left(\lambda_{*}, \infty\right)$, there exist $m_{1}(\lambda), m_{2}(\lambda), M_{1}(\lambda)$ and $M_{2}(\lambda)$ such that

$$
0<m_{2}(\lambda)<m_{1}(\lambda)<m_{*}<M_{1}(\lambda)<M_{2}(\lambda)
$$

and

$$
R_{1}\left(m_{1}(\lambda)\right)=R_{1}\left(M_{1}(\lambda)\right)<\lambda<R_{2}\left(m_{2}(\lambda)\right)=R_{2}\left(M_{2}(\lambda)\right) .
$$

Then the proof is complete by the argument similar to those in the proof of Theorem 5 (2).

(5) Since $f_{0}=f_{\infty}=\infty$, it follows that, for $i=1,2, \lim _{m \rightarrow 0} R_{i}(m)=\lim _{m \rightarrow \infty} R_{i}(m)=0$. Let $\lambda^{*}=$ $\max \left\{R_{2}(m): m \in \mathbb{R}_{+}\right\} \in(0, \infty)$ and $m^{*} \in(0, \infty)$ satisfying $R_{2}\left(m^{*}\right)=\lambda^{*}$. Then the proof is complete by the argument similar to those in the proof of Theorem 5 (2).

(6) Let $u$ be a positive solution to problem (1) with $\lambda>0$ and let $\sigma$ be a constant satisfying $u(\sigma)=\|u\|_{\infty}$. Since $f_{0} \in[0, \infty)$ and $f_{\infty} \in[0, \infty)$, there exists $C_{1}>0$ such that $f(s) \leq C_{1} \varphi(s)$ for $s \in \mathbb{R}_{+}$. We only consider the case $\sigma \leq \gamma_{h}$, since the case $\sigma>\gamma_{h}$ can be dealt in a similar manner. Since $f(u(t)) \leq C_{1} \varphi(u(\sigma))$ for $t \in[0,1]$,

$$
\begin{aligned}
u(\sigma) & =\int_{0}^{\sigma} \frac{1}{c(s)} \varphi^{-1}\left(\frac{1}{d(s)} \int_{s}^{\sigma} \lambda h(\tau) f(u(\tau)) d \tau\right) d s \\
& \leq c_{0}^{-1} \int_{0}^{\gamma_{h}} \varphi^{-1}\left(\int_{s}^{\gamma} h(\tau) d \tau d_{0}^{-1} \lambda C_{1} \varphi(u(\sigma))\right) d s \\
& \leq c_{0}^{-1} h^{*} \varphi^{-1}\left(d_{0}^{-1} \lambda C_{1} \varphi(u(\sigma))\right) \leq c_{0}^{-1} h^{*} \psi_{1}^{-1}\left(d_{0}^{-1} \lambda C_{1}\right) u(\sigma) .
\end{aligned}
$$

Here

$$
h^{*}=\max \left\{\int_{0}^{\gamma_{h}} \psi_{1}^{-1}\left(\int_{s}^{\gamma_{h}} h(\tau) d \tau\right) d s, \int_{\gamma_{h}}^{1} \psi_{1}^{-1}\left(\int_{\gamma_{h}}^{s} h(\tau) d \tau\right) d s\right\}>0
$$

Consequently,

$$
\lambda \geq \frac{d_{0}}{C_{1}} \psi_{1}\left(\frac{c_{0}}{h^{*}}\right)=: \bar{\lambda}
$$

(7) Let $u$ be a positive solution to problem (1) with $\lambda>0$ and let $\sigma$ be a constant satisfying $u(\sigma)=\|u\|_{\infty}$. Since $f_{0} \in(0, \infty]$ and $f_{\infty} \in(0, \infty]$, there exists $\epsilon>0$ such that $f(s)>\epsilon \varphi(s)$ for $s \in \mathbb{R}_{+}$. We only consider the case $\sigma \geq \gamma_{h}$, since the case $\sigma<\gamma_{h}$ can be dealt in a similar manner. Since $u(t) \geq u\left(\gamma_{h}^{1}\right)$ for $t \in\left[\gamma_{h}^{1}, \sigma\right]$,

$$
f(u(t))>\epsilon \varphi\left(u\left(\gamma_{h}^{1}\right)\right) \text { for } t \in\left[\gamma_{h}^{1}, \gamma\right] .
$$

Then

$$
\begin{aligned}
u\left(\gamma_{h}^{1}\right) & =\int_{0}^{\gamma_{h}^{1}} \frac{1}{c(s)} \varphi^{-1}\left(\frac{1}{d(s)} \int_{s}^{\sigma} \lambda h(\tau) f(u(\tau)) d \tau\right) d s \\
& \geq\|c\|_{\infty}^{-1} \int_{0}^{\gamma_{h}^{1}} \varphi^{-1}\left(\int_{\gamma_{h}^{1}}^{\gamma_{h}} h(\tau) d \tau\|d\|_{\infty}^{-1} \lambda \epsilon \varphi\left(u\left(\gamma_{h}^{1}\right)\right)\right) d s \\
& \geq\|c\|_{\infty}^{-1} \gamma_{0} \varphi^{-1}\left(h_{*}\|d\|_{\infty}^{-1} \lambda \epsilon \varphi\left(u\left(\gamma_{h}^{1}\right)\right)\right) \geq\|c\|_{\infty}^{-1} \gamma_{0} \psi_{2}^{-1}\left(h_{*}\|d\|_{\infty}^{-1} \lambda \epsilon\right) u\left(\gamma_{h}^{1}\right) .
\end{aligned}
$$

Here

$$
\gamma_{0}=\min \left\{\gamma_{h^{\prime}}^{1}, 1-\gamma_{h}^{2}\right\}>0 \text { and } h_{*}=\min \left\{\int_{\gamma_{h}^{1}}^{\gamma_{h}} h(\tau) d \tau, \int_{\gamma_{h}}^{\gamma_{h}^{2}} h(\tau) d \tau\right\}>0 .
$$

Consequently,

$$
\lambda \leq \frac{\|d\|_{\infty}}{h_{*} \epsilon} \psi_{2}\left(\frac{\|c\|_{\infty}}{\gamma_{0}}\right)=: \underline{\lambda}
$$


Finally, an example to illustrate the results obtained in this paper is given.

Example 1. Let $\varphi$ be an odd function satisfying either

$$
\text { (i) } \varphi(x)=\frac{x^{2}}{1+x} \text { or }(\text { ii }) \varphi(x)=x+x^{2} \text { for } x \geq 0 \text {. }
$$

Then it is easy to check that $(A)$ is satisfied for

$$
\psi_{1}(y)=\min \left\{y, y^{2}\right\} \text { and } \psi_{2}(y)=\max \left\{y, y^{2}\right\} .
$$

Define $h:(0,1) \rightarrow \mathbb{R}_{+}$by

$$
h(t)=0 \text { for } t \in\left[0, \frac{1}{16}\right] \text { and } h(t)=\left(t-\frac{1}{16}\right)(1-t)^{-a} \text { for } t \in\left(\frac{1}{16}, 1\right) .
$$

Then, since $\psi_{1}^{-1}(s)=s$ for all $s \geq 1, h \in \mathcal{H}_{\psi_{1}} \backslash L^{1}(0,1)$ for any $a \in[1,2)$. Also,

$$
\alpha_{h}=\bar{\beta}_{h}=\frac{1}{16}, \beta_{h}=\bar{\alpha}_{h}=1, \gamma_{h}^{1}=\frac{19}{64}, \gamma_{h}^{2}=\frac{49}{64} \text { and } \gamma_{h}=\frac{17}{32} \text {. }
$$

Let $c, d$ be any positive continuous functions on $[0,1]$. Then $\rho_{1}, \rho_{h}, A_{1}$ and $A_{2}$ are well defined in $(0, \infty)$. Define $f: \mathbb{R}_{+} \rightarrow \mathbb{R}_{+}$by

$$
f(s)= \begin{cases}(\varphi(s))^{2}, & \text { for } s \in[0,1] \\ (\varphi(1))^{\frac{3}{2}}(\varphi(s))^{\frac{1}{2},} & \text { fors } \in\left(1, M_{2}\right] \\ (\varphi(1))^{\frac{3}{2}}\left(\varphi\left(M_{2}\right)\right)^{-\frac{3}{2}}(\varphi(s))^{2}, & \text { for } \in\left(M_{2}, \infty\right) .\end{cases}
$$

Here $M_{2}$ is a fixed constant satisfying

$$
M_{2}>\max \left\{\frac{1}{\rho_{h}}, \varphi^{-1}\left(\frac{1}{\varphi(1)}\left[\varphi\left(\frac{1}{\rho_{h} A_{1}}\right)\right]^{2}\left[\psi_{1}\left(\frac{1}{A_{2}}\right)\right]^{-2}\right)\right\} .
$$

Clearly, $f \in C\left(\mathbb{R}_{+}, \mathbb{R}_{+}\right)$satisfies that $f(s)>0$ for $s>0, f_{0}=0$ and $f_{\infty}=\infty$. Since $f$ is strictly increasing on $\mathbb{R}_{+}$,

$$
f^{*}(m)=f(m) \text { and } f_{*}(m)=f\left(\rho_{h} m\right) \text { for all } m \in \mathbb{R}_{+} .
$$

Then, by (2),

$$
R_{1}(m)=\frac{1}{f\left(\rho_{h} m\right)} \varphi\left(\frac{m}{A_{1}}\right) \text { and } R_{2}(m)=\frac{1}{f(m)} \varphi\left(\frac{m}{A_{2}}\right) \geq \frac{\varphi(m)}{f(m)} \psi_{1}\left(\frac{1}{A_{2}}\right) \text { for } m \in(0, \infty) .
$$

From the choice of $M_{2}$, it follows that

$$
R_{1}\left(\frac{1}{\rho_{h}}\right)=\frac{1}{f(1)} \varphi\left(\frac{1}{\rho_{h} A_{1}}\right)=\frac{1}{[\varphi(1)]^{2}} \varphi\left(\frac{1}{\rho_{h} A_{1}}\right)<\frac{\left[\varphi\left(M_{2}\right)\right]^{\frac{1}{2}}}{[\varphi(1)]^{\frac{3}{2}}} \psi_{1}\left(\frac{1}{A_{2}}\right)=\frac{\varphi\left(M_{2}\right)}{f\left(M_{2}\right)} \psi_{1}\left(\frac{1}{A_{2}}\right) \leq R_{2}\left(M_{2}\right) .
$$

We may choose $m_{2}$ and $M_{1}$ satisfying $0<m_{2}<m_{1}=\rho_{h}^{-1}<M_{2}<M_{1}$ and

$$
R_{1}\left(M_{1}\right) \leq R_{1}\left(\rho_{h}^{-1}\right)<R_{2}\left(M_{2}\right) \leq R_{2}\left(m_{2}\right) ，
$$

since $R_{2}(m) \rightarrow \infty$ as $m \rightarrow 0$ and $R_{1}(m) \rightarrow 0$ as $m \rightarrow \infty$. By Theorems 4 and 5 (1), problem (1) has three positive solutions for $\lambda \in\left(R_{1}\left(\rho_{h}^{-1}\right), R_{2}\left(M_{2}\right)\right)$ and it has a positive solution $u(\lambda)$ for $\lambda \in(0, \infty)$ satisfying that $\lim _{\lambda \rightarrow 0}\|u(\lambda)\|_{\infty}=\infty$ and $\lim _{\lambda \rightarrow \infty}\|u(\lambda)\|_{\infty}=0$. 


\section{Conclusions}

In this work, we studied the existence and nonexistence of positive solutions to problem (1). In Theorems $2-5$, various sufficient conditions on the nonlinearity $f$ for the existence, nonexistence and multiplicity of positive solutions to problem (1) were given. In particular, Theorem 5 improves on the results in Reference [2], since we do not assume the monotonicity of $d$ and the weight function $h:(0,1) \rightarrow \mathbb{R}_{+}$may not be $L^{1}(0,1)$ and it can be vanished in some subinterval of $(0,1)$.

In Reference [1], the nonlinearity $f=f(t, s)$ should satisfy the condition

$$
f\left(t_{0}, 0\right)>0 \text { for some } t_{0} \in[0,1]
$$

so that the existence of an unbounded solution component was shown and, by examining the shape of the component according to several assumptions on $f$ at $\infty$, the existence, nonexistence and multiplicity of positive solutions were studied. In this case, all nonnegative solutions are positive ones by (11). Compared with the results in Reference [1], the nonlinearity $f=f(s)$ in the present work may have the property $f(0)=0$. Even though the existence of unbounded solution component to problem (1) can be obtained by Reference ([1], Theorem 1), the existence of positive solutions cannot be shown from the solution component, since problem (1) has a trivial solution 0 for every $\lambda \in \mathbb{R}_{+}$, provided $f(0)=0$. Thus, the fixed point index theory was used in order to prove the main results (Theorems 2-5).

In the present work, the problem with Dirichlet boundary conditions was considered. As an extension of the results in this paper, similar results for the problem with nonlocal boundary conditions is expected. The existence, nonexistence and multiplicity of positive solutions to problem with nonlocal boundary conditions will be discussed for future work.

Funding: This research was supported by Basic Science Research Program through the National Research Foundation of Korea(NRF) funded by the Ministry of Education(2017R1D1A1B03035623).

Acknowledgments: The author would like to thank the anonymous reviewers for their helpful remarks.

Conflicts of Interest: The author declares no conflict of interest.

\section{References}

1. Jeong, J.; Kim, C.G. Existence of Positive Solutions to Singular Boundary Value Problems Involving $\varphi$-Laplacian. Mathematics 2019, 7, 654. [CrossRef]

2. Wang, H. On the structure of positive radial solutions for quasilinear equations in annular domains. Adv. Differ. Equ. 2003, 8, 111-128.

3. Bandle, C.; Coffman, C.V.; Marcus, M. Nonlinear elliptic problems in annular domains. J. Differ. Equ. 1987, 69, 322-345. [CrossRef]

4. Graef, J.R.; Yang, B. Boundary value problems for second order nonlinear ordinary differential equations. Commun. Appl. Anal. 2002, 6, 273-288.

5. Henderson, J.; Wang, H. Positive solutions for nonlinear eigenvalue problems. J. Math. Anal. Appl. 1997, 208, 252-259. [CrossRef]

6. Iturriaga, L.; Massa, E.; Sánchez, J.; Ubilla, P. Positive solutions for an elliptic equation in an annulus with a superlinear nonlinearity with zeros. Math. Nachr. 2014, 287, 1131-1141. [CrossRef]

7. Lan, K.Q. Multiple positive solutions of semilinear differential equations with singularities. J. Lond. Math. Soc. 2001, 63, 690-704. [CrossRef]

8. Lan, K.; Webb, J.R.L. Positive solutions of semilinear differential equations with singularities. J. Differ. Equ. 1998, 148, 407-421. [CrossRef]

9. Lin, S.S. Positive radial solutions and nonradial bifurcation for semilinear elliptic equations in annular domains. J. Differ. Equ. 1990, 86, 367-391. [CrossRef]

10. Agarwal, R.P.; Lü, H.; O’Regan, D. Eigenvalues and the one-dimensional p-Laplacian. J. Math. Anal. Appl. 2002, 266, 383-400. [CrossRef] 
11. del Pino, M.; Elgueta, M.; Manásevich, R. A homotopic deformation along $p$ of a Leray-Schauder degree result and existence for $\left(\left|u^{\prime}\right|^{p-2} u^{\prime}\right)^{\prime}+f(t, u)=0, u(0)=u(T)=0, p>1$. J. Differ. Equ. 1989, 80, 1-13. [CrossRef]

12. Kim, C.G. Existence of positive solutions for singular boundary value problems involving the one-dimensional p-Laplacian. Nonlinear Anal. 2009, 70, 4259-4267. [CrossRef]

13. Kim, C.G. The three-solutions theorem for $p$-Laplacian boundary value problems. Nonlinear Anal. 2012, 75, 924-931. [CrossRef]

14. Rynne, B.P. Exact multiplicity and stability of solutions of a 1-dimensional, $p$-Laplacian problem with positive convex nonlinearity. Nonlinear Anal. 2019, 183, 271-283. [CrossRef]

15. Sim, I.; Tanaka, S. Three positive solutions for one-dimensional $p$-Laplacian problem with sign-changing weight. Appl. Math. Lett. 2015, 49, 42-50. [CrossRef]

16. Wang, J. The existence of positive solutions for the one-dimensional p-Laplacian. Proc. Am. Math. Soc. 1997, 125, 2275-2283. [CrossRef]

17. Wang, J.; Gao, W. A singular boundary value problem for the one-dimensional p-Laplacian. J. Math. Anal. Appl. 1996, 201, 851-866. [CrossRef]

18. Yang, G.; Li, Z. Positive Solutions of One-Dimensional p-Laplacian Problems with Superlinearity. Symmetry 2018, 10, 363. [CrossRef]

19. Shivaji, R.; Sim, I.; Son, B. A uniqueness result for a semipositone $p$-Laplacian problem on the exterior of a ball. J. Math. Anal. Appl. 2017, 445, 459-475. [CrossRef]

20. $\mathrm{Xu}, \mathrm{X}$. A new existence result for the boundary value problem of $p$-Laplacian equations with sign-changing weights. Proc. Indian Acad. Sci. Math. Sci. 2019, 129, 47. [CrossRef]

21. Bai, D.; Chen, Y. Three positive solutions for a generalized Laplacian boundary value problem with a parameter. Appl. Math. Comput. 2013, 219, 4782-4788. [CrossRef]

22. Lee, Y.H.; Xu, X. Existence and Multiplicity Results for Generalized Laplacian Problems with a Parameter. Bull. Malays. Math. Sci. Soc. 2018. [CrossRef]

23. Kaufmann, U.; Milne, L. Positive solutions for nonlinear problems involving the one-dimensional $\varphi$-Laplacian. J. Math. Anal. Appl. 2018, 461, 24-37. [CrossRef]

24. Sim, I. On the existence of nodal solutions for singular one-dimensional $\phi$-Laplacian problem with asymptotic condition. Commun. Pure Appl. Anal. 2008, 7, 905-923. [CrossRef]

25. García-Huidobro, M.; Manásevich, R.; Ward, J.R. Positive solutions for equations and systems with p-Laplace-like operators. Adv. Differ. Equ. 2009, 14, 401-432.

26. Kaufmann, U.; Milne, L. On one-dimensional superlinear indefinite problems involving the $\phi$-Laplacian. J. Fixed Point Theory Appl. 2018, 20, 134. [CrossRef]

27. Kim, C.G.; Lee, Y.H. Existence of multiple positive solutions for $p$-Laplacian problems with a general indefinite weight. Commun. Contemp. Math. 2008, 10, 337-362. [CrossRef]

28. Deimling, K. Nonlinear Functional Analysis; Springer: Berlin, Germany, 1985.

29. Guo, D.J.; Lakshmikantham, V. Nonlinear Problems in Abstract Cones; Notes and Reports in Mathematics in Science and Engineering; Academic Press, Inc.: Boston, MA, USA, 1988; Volume 5.

(c) 2019 by the author. Licensee MDPI, Basel, Switzerland. This article is an open access article distributed under the terms and conditions of the Creative Commons Attribution (CC BY) license (http://creativecommons.org/licenses/by/4.0/). 